PROCEEDINGS OF THE

AMERICAN MATHEMATICAL SOCIETY

Volume 131, Number 2, Pages 425-432

S 0002-9939(02)06470-5

Article electronically published on September 25, 2002

\title{
TRIANGULATIONS AND HOMOLOGY OF RIEMANN SURFACES
}

\author{
PETER BUSER AND MIKA SEPPÄLÄ
}

(Communicated by Ronald A. Fintushel)

\begin{abstract}
We derive an algorithmic way to pass from a triangulation to a homology basis of a (Riemann) surface. The procedure will work for any surfaces with finite triangulations. We will apply this construction to Riemann surfaces to show that every compact hyperbolic Riemann surface $X$ has a homology basis consisting of curves whose lengths are bounded linearly by the genus $g$ of $X$ and by the homological systole.

This work got started by comments presented by Y. Imayoshi in his lecture at the 37th Taniguchi Symposium which took place in Katinkulta near Kajaani, Finland, in 1995.
\end{abstract}

\section{INTRODUCTION}

The topology of a surface $X$ is determined by the combinatorial structure of any of its triangulations. Here we apply that observation to the study of the homology of a compact Riemann surface of genus $g, g>1$.

Given a triangulated Riemann surface $X$ it appears possible that a homology basis for $X$ can be built out of closed curves that are unions of edges of the triangles. The formation of these curves may be quite complicated. It also appears possible that the curves forming a homology basis can be chosen in such a way that any edge of any triangle of the triangulation appears at most twice in any given curve of the homology basis. This means that every curve in the homology basis consists of at most $2 n_{1}=3 n_{2}$ edges. Here $n_{1}$ is the number of edges of the triangulation and $n_{2}$ the number of triangles.

Now if the Riemann surface $X$ does not have short geodesics, then one can always find a triangulation of $X$ for which the lengths of the edges are universally bounded and for which $n_{2} \leq C g$, where $C$ is a constant that will be specified below. This means that a Riemann surface which does not have short geodesics always carries a homology basis consisting of curves whose lengths are linearly bounded by the genus.

In order to prove the above we derive an algorithmic way to obtain an explicit canonical homology basis for a triangulated Riemann surface $X$. By a canonical homology basis we mean any family of homologically non-trivial simple closed curves $\alpha_{1}, \beta_{1}, \ldots, \alpha_{g}, \beta_{g}$ on $X$ such that each $\alpha_{i}$ intersects $\beta_{i}$ in exactly one point and such

Received by the editors April 23, 2001 and, in revised form, July 11, 2001.

2000 Mathematics Subject Classification. Primary 30F45; Secondary 57M20.

Key words and phrases. Triangulation, homology, Riemann surfaces.

The research of the first author was supported by the Swiss National Research Foundation. 
that no other intersections occur. The method leads to a homology basis whose curves are unions of at most $n_{2} / 2$ edges of the triangulation.

The construction is general and can be applied to any topological surface. One complication in the process is to make sure that we find curves satisfying the required intersection property.

As indicated above, we apply this construction to find a short canonical homology basis for a compact Riemann surface together with the Poincaré metric of constant curvature -1 . Such homology bases are important for numerical computations on Riemann surfaces.

In [6] we have shown that given a family $\mathcal{P}$ of simple closed geodesics on $X$ that decomposes $X$ into three-holed spheres then one can find such a canonical homology basis where $\alpha_{1}, \ldots, \alpha_{g}$ belong to $\mathcal{P}$ and where any $\beta_{i}$ intersects $\mathcal{P}$ at most $2 g-2$ times. The bound $2 g-2$ is the best one possible. If we combine this with Bers' partitioning theorem $([2], 5])$, then we also get an upper bound of the lengths of $\alpha_{1}, \beta_{1}, \ldots, \alpha_{g}, \beta_{g}$, with respect to the hyperbolic metric. That bound is of order $g^{2}$ and it also depends on the homological systole (see below).

Starting with a suitable triangulation rather than with a pants decomposition we show, as indicated above, that there always exists a canonical homology basis consisting of curves whose lengths are bounded linearly by the genus.

To state the result let us introduce the following terminology. The homological systole $\sigma=\sigma(X)$ is the length of a shortest homologically non-trivial simple closed geodesic $\gamma$ on $X$ (there may be several such curves). Any homology basis must contain at least one curve that intersects $\gamma$ transversely. By the Collar Theorem ([3], [4], [8]), this curve becomes long if the length of $\gamma$ is small. Our result is now as follows.

Theorem 1.1. Let $X$ be a compact Riemann surface of genus $g$ with homological systole $\sigma$. There exists a canonical homology basis on $X$ such that any curve has length

$$
\ell<(g-1)\left(45+6 \operatorname{arcsinh}\left(\frac{2}{\sigma}\right)\right) .
$$

The constants 45 and 6 are not optimal. We point out, however, that in the example given in 6] any homology basis must contain at least one curve whose length exceeds $(g-1)\left(2+2 \operatorname{arcsinh}\left(\frac{2}{\sigma}\right)\right)$. For convenience we repeat this example at the end of the paper. Hence the result obtained here is of the best possible order of magnitude.

\section{From triangulations to homology}

Consider a triangulation $\mathcal{T}$ of a surface $X$. Let $V$ be the set of vertices of $\mathcal{T}$, and let $\mathcal{G}$ be the graph whose vertex set is $V$ and whose edges are the edges occurring in $\mathcal{T}$. On $\mathcal{G}$ we select a spanning tree, i.e. a connected subgraph $\Gamma$ as shown in Figure 1which has the same vertices as $\mathcal{G}$ but which is such that no non-trivial closed edge path occurs.

A spanning tree can be obtained by the following procedure. First one selects an edge which does not separate $\mathcal{G}$ into two parts, and erases this edge from $\mathcal{G}$. If on the remaining graph there is again a non-separating edge, then one erases this one also, and so on. Note that $\Gamma$ has $|V|-1$ edges where $|V|$ is the number of vertices of $V$. 


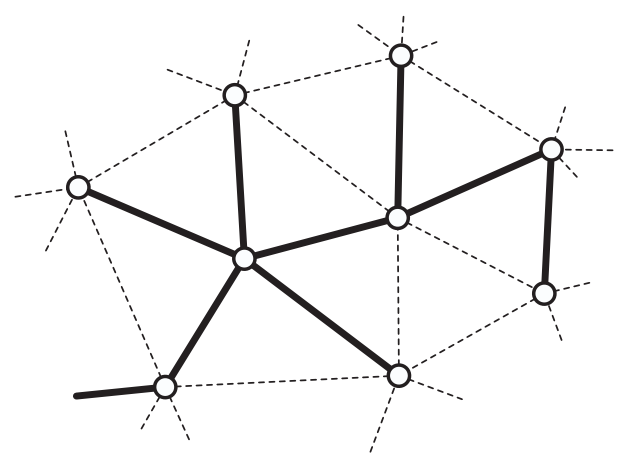

Figure 1. A spanning tree

In the next step we construct a fundamental polygon for $X$ using the triangulation. For this we cut $X$ open along all the edges of $\Gamma$. Since $\Gamma$ is a tree this produces a connected surface $X_{g}$ of signature $(g, 1)$. On $X_{g}$ the triangulation now has the property that all vertices lie on the boundary. Let us denote by $E$ the set of all edges of $\mathcal{G}$ which are not subsets of the boundary of $X_{g}$. We now iteratively cut open $X_{g}$ along certain edges of $E$ until we get the desired polygon. This can be done by the following procedure.

First we look for an edge $u \in E$ which does not separate $X_{g}$ into two parts. Such an edge exists because $X_{g}$ has Euler characteristic $\chi=2-2 g-1$. We recall that $u$ has both end points on the boundary of $X_{g}$. Cutting $X_{g}$ open along $u$ we therefore obtain a connected surface $X_{g}^{\prime}$ with Euler characteristic $\chi^{\prime}=\chi+1$. Since $X_{g}$ is orientable, $X_{g}^{\prime}$ has two connected boundary components, say $\omega^{\prime}$ and $\omega^{\prime \prime}$. Let us denote by $u^{\prime}$ and $u^{\prime \prime}$ the two copies of $u$ on the boundary of $X_{g}^{\prime}$, the notation being such that $u^{\prime} \subset \omega^{\prime}$ and $u^{\prime \prime} \subset \omega^{\prime \prime}$.

Next, we look for an edge $v \in E$ that connects $\omega^{\prime}$ with $\omega^{\prime \prime}$. To see that such an edge exists we consider a continuous curve $c$ going from $\omega^{\prime}$ to $\omega^{\prime \prime}$. The initial point of $c$ lies on a triangle which has a vertex on $\omega^{\prime}$; the end point of $c$ lies on a triangle which has a vertex on $\omega^{\prime \prime}$. If the initial triangle has a vertex on $\omega^{\prime \prime}$ also, we are done. Otherwise we go along $c$ until we reach for the first time a triangle which does not have all three vertices on $\omega^{\prime}$. This triangle shares a vertex with the preceding one and, therefore, has an edge $v$ going from $\omega^{\prime}$ to $\omega^{\prime \prime}$.

Cutting $X_{g}^{\prime}$ open along $v$ we obtain a connected surface $X_{g-1}$ which now has again a connected boundary, say $\omega$, and which has Euler characteristic $\chi^{\prime}+1$. Let us denote by $v^{\prime}$ and $v^{\prime \prime}$ the two copies of $v$ on $\omega$. Note that along $\omega$ the pair $v^{\prime}, v^{\prime \prime}$ is separated by the pair $u^{\prime}, u^{\prime \prime}$.

Since $X_{g-1}$ has signature $(g-1,1)$ we can repeat the procedure to obtain successively $X_{g-2}, X_{g-3}$, etc. until the fundamental polygon, $X_{0}$, is reached after $g$ steps.

For the convenience of later reference we remark next that the triangulation of $X$ induces a triangulation of $X_{0}$ with all the vertices on the boundary of $X_{0}$. The following observation now follows from the fact that $X_{0}$ can be modelled as a polygon in the plane. 


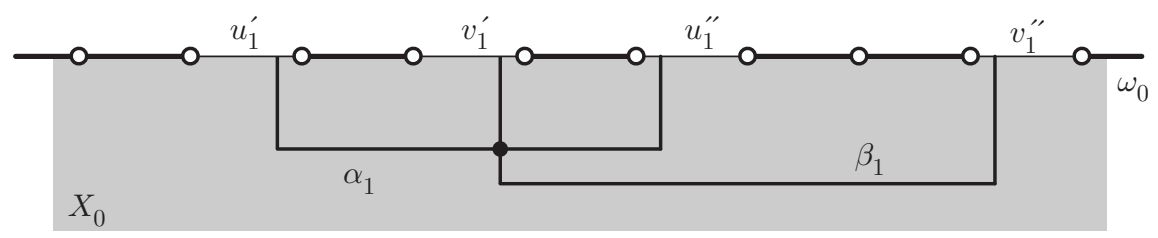

Figure 2. Constructing the homology basis

Lemma 2.1. The boundary of the fundamental polygon $X_{0}$ for the Riemann surface $X$ consists of $n_{2}+2$ edges of the triangulation of $X$.

The construction of the homology basis is now fairly easy. Starting on $X_{0}$ we let $u_{1}^{\prime}, u_{1}^{\prime \prime}$ and $v_{1}^{\prime}, v_{1}^{\prime \prime}$ be the sides on the boundary $\omega_{0}$ of $X_{0}$ obtained when $X_{1}$ was cut open. We draw a curve $\alpha_{1}$ in a small neighborhood of $\omega_{0}$ from a point of $u_{1}^{\prime}$ to an equivalent point of $u_{1}^{\prime \prime}$ (with respect to the pasting) and then a similar curve $\beta_{1}$ from some point of $v_{1}^{\prime}$ to the corresponding point of $v_{1}^{\prime \prime}$ as shown in Figure 2 Then we paste the pairs $u_{1}^{\prime}, u_{1}^{\prime \prime}$ and $v_{1}^{\prime}, v_{1}^{\prime \prime}$ together again to obtain $X_{1}$ on which $\alpha_{1}$ and $\beta_{1}$ are now a pair of simple closed curves intersecting each other in exactly one point.

In the same way we may now continue on $X_{1}$, then on $X_{2}$, and so on. Since on each $X_{k}$ the curves $\alpha_{k+1}, \beta_{k+1}$ can be drawn arbitrarily close to the boundary they do not intersect the preceding curves. Hence, the curve system obtained in this way has the required intersection properties.

\section{Length estimates for surfaces Without ShORT GeOdesics}

To find a short homology basis using the construction of Section 2 we need to start with a triangulation whose triangles are not too small. Such a triangulation can be found if the Riemann surface $X$ does not have short closed geodesic curves. We use the following result from [4, pp. 116-121], respectively [7, which goes back to [9], [10] and others.

Lemma 3.1. Let $X$ be a compact Riemann surface of genus $g \geq 2$ such that the length of the shortest closed geodesic is greater than or equal to $\ln 4$. Then there exists a geodesic triangulation $\mathcal{T}$ on $X$ with the following properties:

(1) All triangles of $\mathcal{T}$ have sidelengths between $\ln 2$ and $2 \ln 2$.

(2) The number of triangles in $\mathcal{T}$ is at most $64(g-1)$.

(The statement for $\mathcal{T}$ in 4 is that the triangles have area $\geq 0.196$. Since $X$ has area $4 \pi(g-1)$ this yields $(2)$.)

As a preparation for the length estimates observe first that in drawing $\alpha_{1}$ and $\beta_{1}$ in Section 2 we have two choices for the directions along $\omega_{0}$. By choosing them properly we can achieve that the curves have lengths $<\frac{1}{2} \ell\left(\omega_{0}\right)$. Likewise, we may choose the directions such that $\alpha_{1}$, respectively $\beta_{1}$, is homotopic to an edge path of $\mathcal{G}$ consisting of at most $\frac{1}{2}\left|\omega_{0}\right|-1$ edges, where $\left|\omega_{0}\right|$ is the number of sides of $\omega_{0}$.

For Riemann surfaces $X$ of genus $g, g \geq 2$, without short geodesics we now have the following result.

Theorem 3.2. Assume that the Riemann surface $X$ has no geodesics shorter than $\ln 4$. Then it has a canonical homology basis consisting of simple closed curves of length less than $45(g-1)$. 
Observe that this result implies Theorem 1.1 for surfaces without short (closed) geodesics.

Proof. To estimate the lengths we note that $X_{0}$ is triangulated with $n_{2} \leq 64(g-1)$ triangles. By Lemma 2.1 the boundary $\omega_{0}$ consists of $n_{2}+2$ edges. The boundaries of the $X_{k}$ have fewer edges. As any edge has length $\leq \ln 4$, all $\alpha_{k}$ and $\beta_{k}$ have, by the remarks preceding the lemma, length $\leq \ln 4\left(\frac{1}{2}\left|\omega_{0}\right|-1\right)<45(g-1)$.

This proves Theorem 1.1 for surfaces without short geodesics. In this proof, the fact that the decomposition of $X$ consisted of triangles was not used in an essential way and only played a role in the estimate of the number of edges. If we use any other cell decomposition $\mathcal{D}$ instead, say with $n_{2}$ cells and $n_{1}$ edges, then the proof works as well. The change in the arithmetic is that now the boundary $\omega_{0}$ of $X_{0}$ has $2\left(n_{1}-n_{2}+1\right)$ edges. Hence, we have the following combinatorial result, where we include the case $g=1$.

Theorem 3.3. Let $\mathcal{D}$ be a cell decomposition of a compact orientable surface $M$ of genus $g \geq 1$ with $n_{2}$ cells and $n_{1}$ edges. Then there exists a canonical homology basis for $M$ such that any curve in this basis is homotopic to an edge path of $\mathcal{D}$ having at most $n_{1}-n_{2}$ edges.

\section{The general CASE}

In the neighborhood of a short geodesic the triangulation of the preceding section cannot be applied, and we need a slightly different cell decomposition of $X$.

Let $\gamma$ be a simple closed short geodesic of $X$ and consider the following set known as the collar around $\gamma$,

$$
\mathcal{C}_{\gamma}=\left\{x \in X \mid \operatorname{dist}(x, \gamma)<w_{\gamma}\right\},
$$

where $w_{\gamma}$, the width of the collar, is defined by the condition $\sinh w_{\gamma} \sinh \frac{1}{2} \ell(\gamma)=1$. By the collar theorem $([8],[3],[4]), \mathrm{C}_{\gamma}$ is a topological cylinder. Furthermore, any closed geodesic intersecting $\gamma$ transversely must cross $\mathcal{C}_{\gamma}$ from one end to the other and therefore has length at least $2 w_{\gamma}$. As short geodesics have length $\leq \ln 4$, this implies that the short geodesics are pairwise disjoint. Moreover, also by the collar theorem, for any distinct short geodesics $\gamma, \gamma^{\prime}$ the collars $\mathcal{C}_{\gamma}$ and $\mathcal{C}_{\gamma^{\prime}}$ are disjoint.

For any short $\gamma$ a domain $R_{\gamma} \subset \mathcal{C}_{\gamma}$ as shown in Figure 3 will be used which is defined as follows. The four vertices $A, A^{\prime}, B, B^{\prime}$ have distance $\frac{1}{2} \ln 2$ from the boundary of $\mathcal{C}_{\gamma}$; the geodesic segments in $\mathcal{C}_{\gamma}$ from $A$ to $A^{\prime}$ and from $B$ to $B^{\prime}$ intersect $\gamma$ orthogonally and in opposite points. The two minimal geodesics from $A$ to $B$ form a simple closed curve $c_{\gamma}$ homotopic to $\gamma$; the minimal geodesics from $A^{\prime}$ to $B^{\prime}$ form a similar curve $c_{\gamma}^{\prime}$. Now $R_{\gamma}$ is the cylindrical domain bounded by $c_{\gamma}$ and $c_{\gamma}^{\prime}$.

If $R_{\gamma}$ is cut open along the geodesic segment $a_{\gamma}$ from $A$ to $A^{\prime}$, then it becomes a geodesic hexagon.

It is shown in [4 p. 116] that $X$ with the interiors of all $R_{\gamma}$ removed can be triangulated such that properties (1), (2) as in Lemma 3.1 hold. This allows us to use the procedure of the preceding section with only minor modifications. First of all we want to avoid intersections with the separating short geodesics. To this end we remove, for any short separating geodesic $\gamma$, the interior of $R_{\gamma}$ from $X$. The resulting surface falls into connected components $S^{1}, \ldots, S^{p}$ with signatures 
$\left(h_{i}, m_{i}\right)$ such that $h_{1}+\cdots+h_{p}=g$, and we now consider one of them, say $S$, with signature $(h, m)$.

Let $c_{1}, \ldots, c_{m}$ be the boundary components of $S$, and let $\gamma_{1}, \ldots, \gamma_{n}$ be the nonseparating short geodesics of $S$. We triangulate the closure of $S \backslash\left(R_{\gamma_{1}} \cup \cdots \cup R_{\gamma_{n}}\right)$ as mentioned above with triangles of sidelengths between $\ln 2$ and $\ln 4$. Next we let $\mathcal{G}$ be the graph whose vertices are the vertices of the triangulation and whose edges are the edges of the triangulation plus the geodesic arcs $a_{\gamma_{1}}, \ldots, a_{\gamma_{n}}$ which cut the $R_{\gamma_{1}}, \ldots, R_{\gamma_{n}}$ into hexagons (see Figure 3 ). Since $S$ has boundary, the spanning tree as used in the preceding section needs a minor modification. We take a connected subgraph $\Gamma$ of $\mathcal{G}$ which has the following properties instead:

(1) any vertex of $\mathcal{G}$ is a vertex of $\Gamma$;

(2) any edge of $\mathcal{G}$ that lies on the boundary of $S$ is an edge of $\Gamma$;

(3) any edge of $\Gamma$ that does not lie on the boundary of $S$ separates $\Gamma$ into two parts.

This is almost a tree, in fact, $\Gamma$ becomes a tree if the cycles $c_{1}, \ldots, c_{m}$ are removed from it.

Cutting $S$ open along the edges of $\Gamma$ not lying on the boundary we obtain a connected surface $S_{h}$ which has only one boundary component. Moreover, the triangles and hexagons yield a cell decomposition of $S_{h}$ all of whose vertices lie on the boundary. Hence, the construction of the preceding section can be carried out on $S_{h}$. As $S_{h}$ has signature $(h, 1)$ we obtain a homology basis $\alpha_{1}, \beta_{1}, \ldots, \alpha_{h}, \beta_{h}$ (modulo the boundary) of $S$.

The bases of $S=S^{1}, \ldots, S^{p}$ taken together yield our homology basis for $X$. To estimate the length of a curve $\eta$ in this basis let us assume that $X$ has $q$ non-separating short geodesics. These are pairwise non-homotopic, and therefore $q \leq 3 g-3$. The domains $R_{\gamma}$ around these geodesics are pairwise disjoint and by [4] pp. 116-117], each of them has surface area larger than 2 . This implies that the number $t$ of all triangles in the cell decomposition of $X$ satisfies $t \leq 64(g-1)-10 q$.

Now consider again the above component $S$ and let $S_{0}$ be the fundamental polygon obtained in the final step of opening it up. The part of the cell decomposition that lies on $S_{0}$ consists of $n_{2}^{\prime} \leq t$ triangles and $q^{\prime} \leq q$ hexagons. As all vertices of this decomposition lie on the boundary of $S_{0}$, the number of edges in the interior of $S_{0}$ is $n_{2}^{\prime}-1$. Each hexagon is obtained by cutting open some $R_{\gamma}$ along the arc $a_{\gamma}$; it has four sides of length $\leq \ln 4$ belonging to the boundary of $R_{\gamma}$, plus two "long" sides corresponding to $a_{\gamma}$. The long sides are shorter than $2 w_{\gamma}$, where in turn $w_{\gamma} \leq \operatorname{arcsinh}\left(\frac{2}{\sigma}\right)$. By construction, all long sides lie on the boundary of $S_{0}$. Hence, the boundary of $S_{0}$ has $2 q^{\prime}$ long sides and $3 n_{2}^{\prime}+4 q^{\prime}-2\left(n_{2}^{\prime}-1\right)$ triangle sides. Reviewing all inequalities we get the following estimate for any curve $\eta$ in

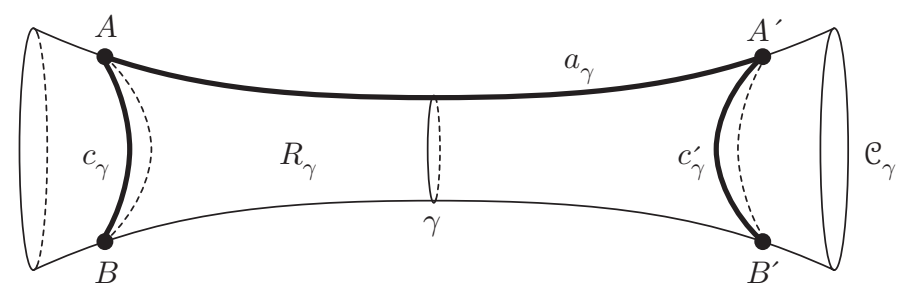

Figure 3. Collar around $\gamma$ 


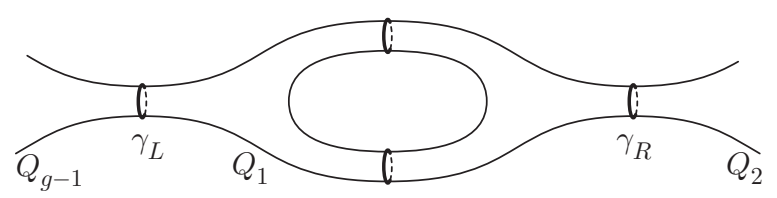

Figure 4. A surface of genus $g$

our homology basis:

$$
\ell(\eta) \leq(32(g-1)-3 q) \ln 4+2 q \operatorname{arcsinh}\left(\frac{2}{\sigma}\right) .
$$

As $q \leq 3 g-3$, this completes the proof of Theorem 1.1

Example 4.2. For comparison we briefly review here the example given in [6]. The surface, $N$, is obtained by pasting together $g-1$ copies $\Omega_{1}, \ldots, \Omega_{g-1}$ of a building block $\Omega$ of signature $(1,2)$ as shown in Figure 1 where $\Omega$ itself is obtained by pasting together two pairs of pants with boundary geodesics of equal lengths $\varepsilon$. By hyperbolic trigonometry, the distance between the two boundary geodesics of $\Omega=\Omega_{1}$ equals

$$
\operatorname{dist}\left(\gamma_{L}, \gamma_{R}\right)=4 \operatorname{arcsinh} \frac{\cosh (\varepsilon / 4)}{\sinh (\varepsilon / 2)} .
$$

On $N$, the geodesic $\gamma_{L}$ is non-separating. Hence, if $\mathcal{B}$ is a canonical homology basis for $N$, then $\mathcal{B}$ contains at least one curve $\beta$ such that $\gamma_{L}$ and $\beta$ have non-zero intersection number. If we cut open $N$ along $\gamma_{L}$, then on the resulting surface $N^{\prime}$ there must be an arc of $\beta$ connecting the two boundary components of $N^{\prime}$ with each other. This implies that $\ell(\beta) \geq(g-1) \operatorname{dist}\left(\gamma_{L}, \gamma_{R}\right)$.

For small values of $\varepsilon$ we have $\varepsilon=\sigma=\sigma(N)$ and the lower bound for $\ell(\beta)$ is approximately $4(g-1) \operatorname{arcsinh}\left(\frac{2}{\sigma}\right)$. If we take $\varepsilon=\ln 4$, then $N$ has no short geodesics and $\ell(\beta)>4.58(g-1)$.

\section{REFERENCES}

[1] Lipman Bers. Finite dimensional Teichmüller spaces and generalizations. Bull. Amer. Math. Soc., 5(2):131 - 172, September 1981. MR 82k:32050

[2] Lipman Bers. An Inequality for Riemann Surfaces. In Isaac Chavel and Hersel M. Farkas, editors, Differential Geometry and Complex Analysis, pages 87 - 93. Springer-Verlag, BerlinHeidelberg-New York, 1985. MR 86h:30076

[3] Peter Buser. The collar theorem and examples. Manuscripta Math., 25:349-357, 1978. MR 80h:53046

[4] Peter Buser. Geometry and Spectra of Compact Riemann Surfaces. Birkhäuser Verlag, BaselBoston-New York, 1992. MR 93g:58149

[5] Peter Buser and Mika Seppälä. Symmetric pants decompositions of Riemann surfaces. Duke Math. J., 67(1):39-55, 1992. MR 93i:32026

[6] Peter Buser and Mika Seppälä. Short homology bases and partitions of Riemann surfaces. Topology, to appear.

[7] Peter Buser, Mika Seppälä and Robert Silhol. Triangulations and moduli spaces of Riemann surfaces with group actions. Manuscripta Math., 88:209-224, 1995. MR 96k:32040

[8] Isaac Chavel and Edgar A. Feldman. Cylinders on surfaces. Comment. Math. Helv., 53:439447, 1978. MR 58:12830 
[9] B. Delaunay. Sur la sphére vide. Proceedings of the International Mathematical Congress held in Toronto August 11-16, Toronto: University of Toronto Press, 695-700, 1928.

[10] L. Fejes Tóth. Kreisausfüllungen der hyperbolischen Ebene. Acta Math. Acad. Sci. Hungar., (4):103-110, 1953.

Département de Mathématiques, Ecole Polytechnique Fédérale de Lausanne, CH1007 Lausanne, Switzerland

E-mail address: Peter.Buser@epfl.ch

Department of Mathematics, Florida State University, Tallahassee, Florida 32306

E-mail address: Mika.Seppala@fsu.edu

Current address: Department of Mathematics, University of Helsinki, FIN-00014 Helsinki, Finland

E-mail address: Mika.Seppala@Helsinki.Fi 